RESEARCH NOTES

\title{
AN EXAMPLE OF AN ALMOST HYPERBOLIC HERMITIAN MANIFOLD
}

\author{
CORNELIA-LIVIA BEJAN
}

Seminarul Matematic Unıversitatea "Al. I Cuza"

Iaşı, ROMANIA

LIVIU ORNEA

Faculty of Mathematics University of Bucharest, ROMANIA

(Received March 15, 1995)

\begin{abstract}
The almost hyperbolic Hermitan manifolds introduced in [1] were classified for the first tume in [2]. In this note we construct an example of an almost hyperbolic Hermitian manifold which belongs to a certain class that we study here
\end{abstract}

KEY WORDS AND PHRASES: Hyperbolic Hermitian manifolds, locally conformal hyperbolic Kähler manifolds, hightlike hypersurface

1991 AMS SUBJECT CLASSIFICATION CODES: 53C15;53C50

\section{INTRODUCTION}

We recall now that the almost hyperbolic Hermitian manifolds have been studied by several authors (see the list of references in [3]). It is known that an almost hyperbolic Hermitian (called also parahermitian) manifold is a pseudo-Riemannian manifold $(\mathrm{M}, \mathrm{g})$ endowed with an almost product structure $P$ wich is skew-symmetric with respect to $g$, i.e.:

$$
\mathrm{P}^{2}=\mathrm{Id} ; \mathrm{P} \neq \pm \mathrm{Id} ; \mathrm{g}(\mathrm{PX}, \mathrm{Y})+\mathrm{g}(\mathrm{X}, \mathrm{PY})=0, \forall \mathrm{X}, \mathrm{Y} \in \chi(\mathrm{M}) .
$$

It follows that $M$ has an even dimension, $g$ has the signature $(n, n)$ and the eigenvalues +1 and -1 have the same multiplicty. These manifolds were clasified in [1] with respect to the covariant derivative of the almost symplectic structure $\Omega$, called the symplectic 2-form associated to $(P, g)$ and defined by:

$$
\Omega(X, Y)=g(P X, Y), \forall X, Y \in \chi(M)
$$


$\mathrm{M}$ is called an almost hyperbolic Kähler manifold if $\Omega$ is closed The notion of almost hyperbolic Hermitian manifold was extended in a natural way to the vector bundles in [4]

We define here a certain class of the almost hyperbolic Hermitian manifolds, that is the locally conformal hyperbolic Kähler (abbreviated 1 c.h.K.) manifolds and then we give an example of such a manifold We recall that the locally conformal Kähler manifolds were studied by I.Vaisman [5] and they are particular cases of locally conformal symplectic manifolds, i.e. there is a family $\left(U_{1}, f_{1}\right)_{1 \in !}$ such that $\left(U_{1}\right)_{i \in I}$ is an open covering of $M$ and for each $i \in I, f_{1}$ is a $C^{\infty}$ function on $U_{1}$ such that the form $f_{1} \Omega$ is closed. This implies the existence of a globally defined 1-form $\omega$ on $M$ (called the Lee form) such that

$$
d \Omega=\omega \wedge \Omega \text {. }
$$

When the dimension of $M$ is greater than 2 then the converse holds good, that is the existence of $\omega$ satisfying (1.3) implies the locally conformal symplectic structure.

We say that an almost hyperbolic Hermitian manifold $(\mathrm{M}, \mathrm{P}, \mathrm{g})$ is $1 . \mathrm{c} . \mathrm{h} \mathrm{K}$. If it is locally conformal symplectic and $\mathbf{P}$ is integrable, that is $\mathbf{P}$ is a product structure.

It is easily seen that $\omega$ is closed. If moreover $\omega$ is an exact form, then $(M, P, g)$ is called a globally conformal hyperbolic Kähler manifold.

\section{THE L.C.H.K. MANIFOLDS}

From now on, we take $(\mathrm{M}, \mathrm{P}, \mathrm{g})$ to be a l.c.h $\mathrm{K}$. manifold havng a nowhere zero Lee form $\omega$. Since $\mathrm{g}$ establishes an isomorphism between the tangent and the cotangent bundle, let $\mathrm{A}$ and $\mathrm{B}$ be two vector fields defined by .

$$
g(X, A)=\omega(P X) \text { and } g(X, B)=\omega(X), \forall X \in \chi(M) .
$$

Then we get $A=-P B$ and $g(A, B)=0$.

We recall that $X \in \chi(M)$ is a time-like, space-like or isotropic vector field if $g(X, X)<0,>0,=0$, respectively.

PROPOSITION 1. Under the above notation, we get :

a) $\mathrm{A}$ is a Killing vector field if and only if it is an infinitesimal automorphism of the almost product structure $P$ and in this case $[A, B]=0$;

b) $\mathrm{A}$ is a time-like (resp. space-like) vector field if and only if $\mathrm{B}$ is a space-like (resp. time-like);

c) The following assertions are equivalent : i) $A$ is an isotropic vector field; ii) $B$ is an isotropic vector field; iii) $\Omega(A, B)=0$.

Our aim now is to study when $B$ is a Killing vector field.

Let $K=\{X \in \chi(M) \mid \omega(X)=0\}$ be the null distribution of $\omega$. We remark that $K$ is of dimension

$n-1$ Since $\omega$ is ctosed, it follows that $K$ is involutive.

Case 1.If $B$ is not an isotropic vector field, we get the orthogonal decomposition $T M=K \perp \operatorname{span}\{B\}$ and it follows that $\mathrm{K}$ is a nondegenerate distribution with respect to $\mathrm{g}$.

We say that $B$ is Killing on a submanifold $N \subset M$ if $L_{B}(g / N)=0$, that is $\left(L_{B} g\right)(X, Y)=0$, $\forall \mathrm{X}, \mathrm{Y} \in \chi(\mathrm{N})$ 
PROPOSITION 2. Under the above notations, we suppose $B$ is a nonisotropic vector field. Then any leaf of $\mathrm{K}$ is a semi-Riemannian hypersurface $\mathrm{H}$ of $\mathrm{M}$ which is normal to $\mathrm{B}$. Moreover, $\mathrm{B}$ is Killing on $\mathrm{H}$ if and only if $\mathrm{H}$ is a totally geodesic hypersurface of $\mathrm{M}$.

PROOF. For the study of semi-Riemannian manifolds and submanifolds we mention [6]. The induced connection D of the Levi-Civita connection $\nabla$ of the semi-Riemannian manfold $(\mathrm{M}, \mathrm{g})$ to the sem1Riemannian submanifold $\left(\mathrm{H},\left.\mathrm{g}\right|_{\mathrm{H}}\right)$ of it is also Levi-Civita. From the Gauss formula

$$
\nabla_{X} Y=D_{X} Y+h(X, Y), \forall X, Y \in \chi(H),
$$

$\mathrm{H}$ is said to be totally geodesic if $\mathrm{h}$ vanishes identically. Now, the assertion of Proposition 2 follows from a straightforward calculation

Case 2. If $B$ is an isotropic vector field, then $A, B \in K$

PROPOSITION 3. Under the above notation, there is an isotropic vector field $C$ such that $\omega(C)=1$

PROOF. As $g$ is nondegenerate, there is a vector field $Q$ such that $g(B, Q) \neq 0$ We take $C=a A+b Q$, where $a, b \in \mathbf{R}$ and we impose the following conditions :

$$
\mathrm{g}(\mathrm{B}, \mathrm{C})=1 \text { and } \mathrm{g}(\mathrm{C}, \mathrm{C})=0
$$

to be satisfied. Therefore we obtain :

$$
C=-g(Q, Q) B / 2(g(B, Q))^{2}+Q / g(B, Q) .
$$

Now, we have the direct sum decomposition $T M=K \oplus \operatorname{span}\{C\}$

As a consequence of Proposition 3, we get that span $\{B, C\}$ is nondegenerate with respect to $g$ If we take a supplimentary orthogonal distribution $S$ of $\operatorname{span}\{B, C\}$ in $T M$, then $S$ is a nondegenerate distribution. Since $B$ belongs to $K$ and in the same time is orthogonal to $K$, we obtain the orthogonal decomposition $\mathrm{K}=\mathrm{S} \perp \operatorname{span}\{\mathrm{B}\}$.

Now, by applying the general theory of degenerate submanifolds of semı-Riemannian manifolds studied in [5], we proved :

PROPOSITION 4. Under the above notation, any leaf of $K$ is a degenerate hypersurface $H$ of $M$ which is normal to $\mathrm{B}$ and the distribution $\mathrm{S}$ restricted to $\mathrm{H}$ is just what A.Bejancu called in [7] the screen distribution.

According to the general results of [7] adapted here, if $\nabla$ is the Levi-Civita connection of the semiRiemannian manifold $(M, g)$, then its induced connection $D$ on the degenerate submanifold $(H, g / H)$ is not necesanly Levi-Civita and the following conditions are equivalent : a) $B$ is a Killing vector field; b) $H$ is totally geodesic in $M ; c) D$ is a metric connection; d) $\operatorname{span}\{B\}$ is parallel with respect to $D$

\section{AN EXAMPLE OF A L.C.H.K. MANIFOLD}

We recall from [1] that the pseudo-Euclidean space of dimension $2 n$ and of index $n\left(R_{n}^{2 n},<,>\right)$, where the pseudo-Euclidean metric $<,>$ has the matrix $\left[\begin{array}{cc}-I_{n} & 0 \\ 0 & I_{n}\end{array}\right]$ with respect to the canonical basis of 
$R_{n}^{2 n}$, can be endowed with the hyperbolic Kähler structure $\left(P^{c a n},<,>\right)$, where $P^{c a n}$ has the matnx $\left[\begin{array}{ll}0 & I_{n} \\ I_{n} & 0\end{array}\right]$ with respect to the canonical basis of $R_{n}^{2 n}$. We denote by $\Omega^{\mathrm{cm}}$ its fundamental 2-form

Now, let $H_{n-1}^{2 n-1}(r)=\left\{x \in R_{n}^{2 n} \mid<x, x>=-r^{2}\right\}$ be the pseudohyperbolic space of radius $r>0$, of dimension $2 n-1$ and of index $n-1, n \in\{2,3 \ldots\}$

Let $\mathrm{N}$ be the unit normal time-like vector field [6]

Since $H_{n-1}^{2 n-1}(r)$ is a pseudo-Riemannian submanifold of the hyperbolic Kàhler manifold $\left(R_{n}^{2 n}, P^{c a n},<,>\right)$, we denote by $g$ the restriction of $<,>$ to $H_{n-1}^{2 n-1}(r)$.

We recall now the construction of the almost paracontact metric structure defined in [8] Let us denote by $\mathrm{D}$ the orthogonal distribution with respect to $\mathrm{g}$ of the vector field $\xi=\mathrm{P}^{\mathrm{cm}} \mathrm{N}$. We define a tensor field $F$ of type (1.1) such that $F / D=P^{c a n}$ and $F \xi=0 F$ is well defined since the tangent bundle $\mathrm{T}\left(\mathrm{H}_{n-1}^{2 n-1}(r)\right)$ can be decomposed into the direct sum $\mathrm{T}\left(\mathrm{H}_{\mathrm{n}-1}^{2 n-1}(\mathrm{r})\right)=\mathrm{D} \oplus \operatorname{span}\{\xi\}$ It is easily seen that $(F, \xi, \eta, g)$ is an almost paracontact metric structure, where $\eta$ is an 1 -form defined by $\eta(X)=g(X, \xi)$, $\forall X \in \chi\left(H_{n-1}^{2 n-1}(r)\right)$. Let $\varphi$ be the 2-form defined by $\varphi(X, Y)=g(F X, Y), \forall X, Y \in \chi\left(H_{n-1}^{2 n-1}(r)\right)$ It follows that $\eta$ and $\varphi$ are closed, [8] We recall from [6] that $H_{n-1}^{2 n-1}(r)$ is a totally umbilic submanifold of $R_{n}^{2 n}$, $\mathrm{n} \geq 2$ and we have

$$
h(X, Y)=g(X, Y) H,
$$

where $h$ is the tensor field of the second fundamental form and $\mathrm{H}$ is the vector field of the normal curvature defined by $\mathrm{H}=\mathrm{N} / \mathrm{r}$

Let $S^{1}$ be the unit circle having $d / d t$ as a nowhere zero vector field and let $\Theta$ be the 1-form such that $\Theta(d / d t)=1$ We denote by $\gamma$ the canonical Riemannian structure of $S^{\prime}$

Now, we construct $M=S^{1} \times H_{n-1}^{2 n-1}(r)$, where $r>0, n \in\{2,3, \ldots\}$ and let $G$ be a pseudo-Riemannian structure on $M$ defined by $\left[\begin{array}{ll}\gamma & 0 \\ 0 & \mathrm{~g}\end{array}\right]$.

Moreover, we define on $M$ a tensor field $P$ of type $(1,1)$ such that : $P(d / d t, 0)=(0, \xi)$; $P(0, \xi)=(d / d t, 0) ; P(0, X)=(0, F X), \forall X \in \Gamma(D)$. It follows that $(P, G)$ is an almost hyperbolic Hermitian structure on $\mathrm{M}$, whose associated symplectic 2-form will be denoted by $\Omega$.

Now, we construct an 1-form $\omega$ defined by $\omega_{(p . q)}=\frac{2}{r}\left(\Theta_{p}, 0\right), \forall(p, q) \in M$. By calculation, we get that the relation (1 2) : 4 satisfied and therefore (M,P,G) is an almost hyperbolic Hermitian manifold wich is locally conformal symplectic We remark that since the 1-form $\omega$ is not an exact form, this structure is not globally conformal symplectic. Since in Proposition 4 the integrability of $P$ is not required, its statement can be applied here. 


\section{CONSEQUENCES}

We recall here a relation between the hyperbolic Hermitian structure and the Hermitian structure on vector bundles

THEOREM 5. [4]. Let (E, $\Pi, M)$ be a real vector bundle of rank $2 k$.

(i) If $(\mathrm{P}, \mathrm{g})$ is a hyperbolic Hermitian structure of $\mathrm{E}$, then it induces a Hermitian structure (J.h) and the associated symplectic structures of $(P, g)$ and $(J, h)$ are equal, that is $g(P X, Y)=h(J X, Y), \forall X, Y \in \Gamma(E)$

(ii) Conversely, if $(\mathrm{J}, \mathrm{h})$ is a Hermitian structure of $\mathrm{E}$ with the associated symplectic structure $\Theta$ (defined by $\Theta(X, Y)=h(J X, Y), \forall X, Y \in \Gamma(E))$ and $S$ is a rank $k$ invariant vector subbundle of $E$ with respect to $J$ (that is $\left.S_{p} \cap J S_{p}=\{0\}, p \in M\right)$, then $(J, h)$ induces a hyperbolic Hermitian structure $(P, g)$ of E. If moreover $S$ is Lagrangian with respect to $\Theta$ (that is $\Theta / S=0$ ), then $\Theta$ is also the symplectic structure associated to $(P, g)($ 1.e. $\Theta(X, Y)=g(P X, Y), \forall X, Y \in \Gamma(E))$.

By taking into account the definition of a locally conformal Kähler manifold [5] recalled here in section 1 , it follows

THEOREM 6. Let $M$ be a $2 k$-dimensional manifold, $k>1$

(i) If $(\mathrm{P}, \mathrm{g})$ is a l.c.h K. structure on $\mathrm{M}$, then it induces a locally conformal Kähler structure (J,h) on $\mathbf{M}$

(ii) Conversely, if $(\mathrm{M}, \mathrm{J}, \mathrm{h})$ is a locally conformal Kähler manifold and $\mathrm{S}$ is an anti-invanant distribution of $M$ with respect to $J$ (that is $S_{p} \cap J S_{p}=\{0\}, p \in M$ ) such that $S$ is Lagrangian (i.e. rank $S=$ $k$ and the fundamental 2-form associated to the almost Hermitian structure (J,h) is zero on $S)$, then $(J, h)$ induces a 1 c.h.K. structure $(P, g)$ on $M$.

PROOF. By taking the vector bundle $E$ to be the tangent bundle TM of the manifold $M$, it follows that Theorem 5 establishes the conditions under which an almost hyperbolic Hermitian structure on $M$ induces an almost Hermitian structure and conversely Since the associated symplectic 2-forms are the same, we obtain that the condition (1.3) for the l.c.h.K. structure and for locally conformal Kähler structure are simultaneously satisfied and therefore we complete the proof.

Now, we suppose $(\mathrm{M}, \mathrm{P}, \mathrm{g})$ is a l.c.h.K. manifold with the vector field $\mathrm{B}$ nonisotropic, that is $\left\|\omega_{\mathrm{p}}\right\|=$ $g_{p}(\omega, \omega)=g_{p}(B, B) \neq 0, p \in M$.

We take $u=\omega /\|\omega\|$ and let $U \in \chi(M)$ be the vector field defined by $g(X, U)=u(X), \forall X \in \chi(M)$ We denote $\mathrm{V}=\mathrm{PU}$ and $\mathrm{v}=\mathrm{uP}$.

A new structure on $M$ can be constructed here By a straightforward calculation, we obtain the following :

PROPOSITION 7. Under the above assumption, if $U$ is a time-like (resp. space-like) vector field, then the tensor field $L$ of type $(1,1)$ defined by $L=P \mp u \otimes V \pm v \otimes U$ satisfies $L^{3}-L=0$, which is a special structure studied in [9].Moreover, we get the following relation between the structure $\mathrm{L}$ and the metric $\mathrm{g}$ :

$$
g(L X, L Y)=-g(X, Y) \pm v(X) v(Y) \mp u(X) u(Y), \forall X, Y \in \chi(M) .
$$




\section{REFERENCES}

[1] LIBERMANN, P., Sur le probleme d'équivalence de certaines structures infinttésımales, Ann. Math. 36 (1954), 27-120

[2] BEJAN, C.L., A classification of almost para-Hermitian manifolds, Proc. Conf. Diff. Geom., Dubrovnik, Yugoslavia, 1988 (1989).

[3] BEJAN, C.L., Structuri hiperbolice pe diferite spații fibrate, Ph. D. Thesis, Iaşı (1990)

[4] BEJAN, C.L., The existence problem of hyperbolic structures on vector bundles, Publ. Inst. Mat. Beograd, tome 53(67) (1993), 133-138.

[5] VAISMAN, I., On locally conformal almost Kähler manifolds, Israel, J. Math., vol 24

[6] O'NEILL, Semi-Riemannian geometry, Acad. Press, New York, (1983)

[7] BEJANCU, A., Degenerated hypersurfaces of semi-Riemannıan manifolds, Bul.Inst. Politeh. Iaşı, tom XXXVII (XLI), fasc. 1-4, 13-22

[8] BEJAN, C.L., CR-submanifolds of hyperbolic Hermitian manifolds, Demonstratio Mat., vol.23 (1990), no 2, 335-343

[9] YANO, K., and KON, M., Structures on manifolds, World Scientific, Singapore (1984). 


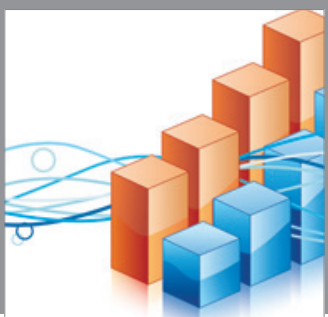

Advances in

Operations Research

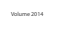

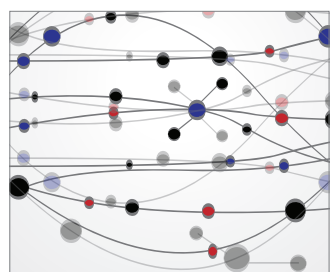

\section{The Scientific} World Journal
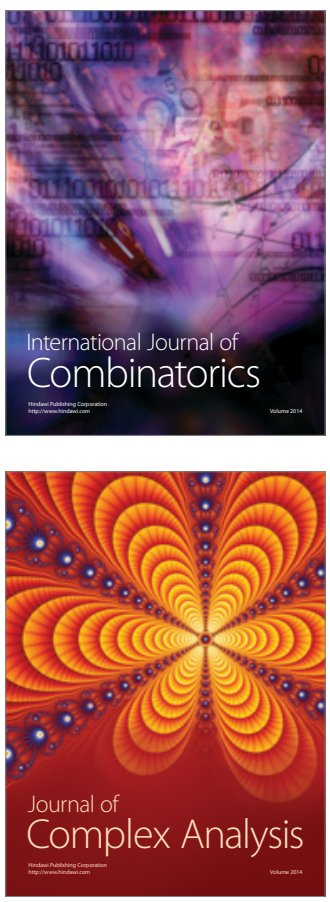

International Journal of

Mathematics and

Mathematical

Sciences
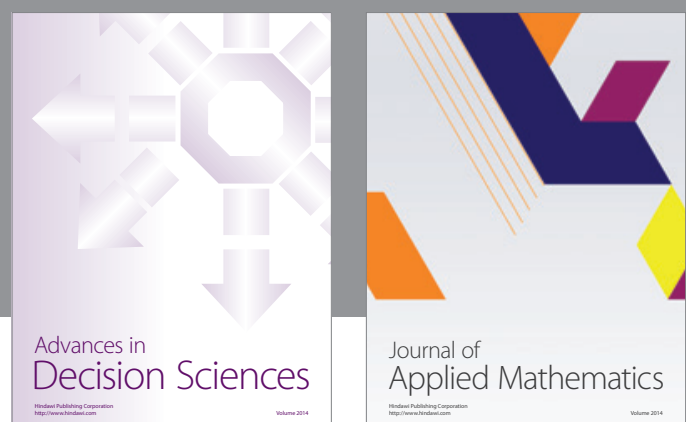

Journal of

Applied Mathematics
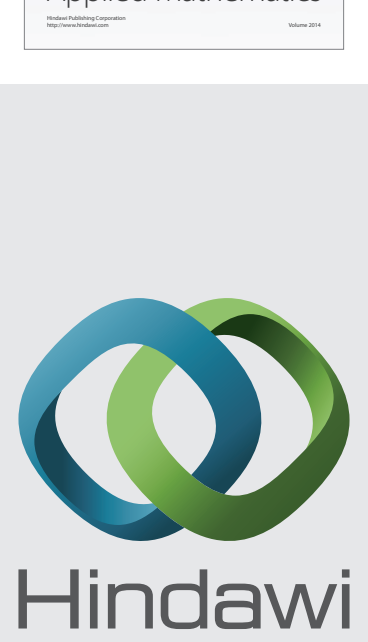

Submit your manuscripts at http://www.hindawi.com
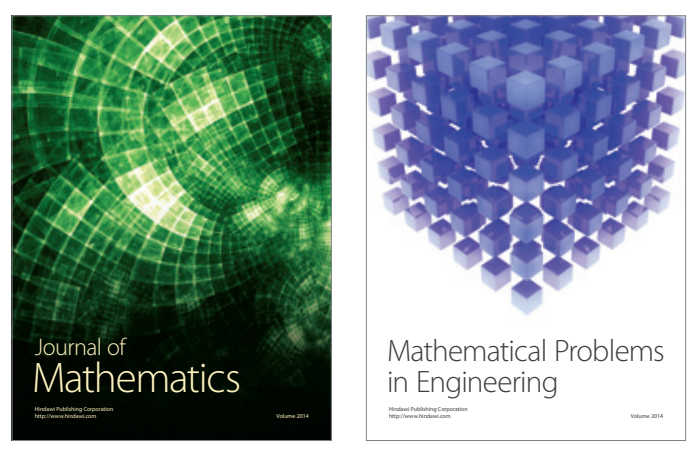

Mathematical Problems in Engineering
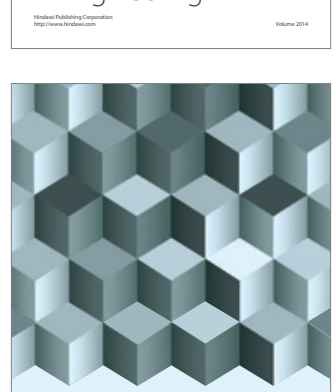

Journal of

Function Spaces
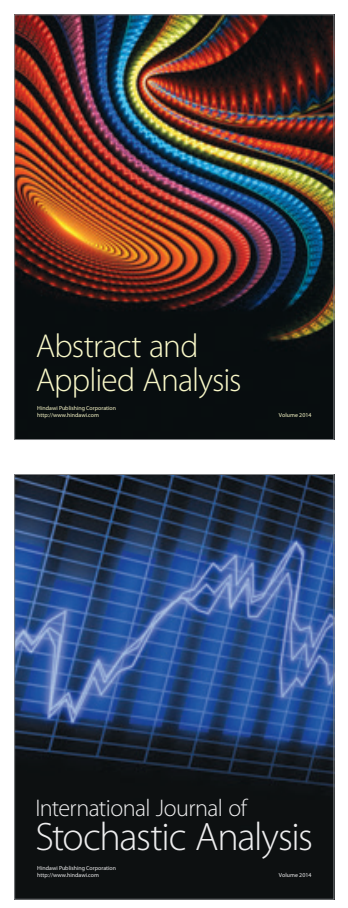

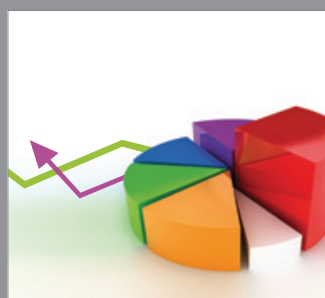

ournal of

Probability and Statistics

Promensencen
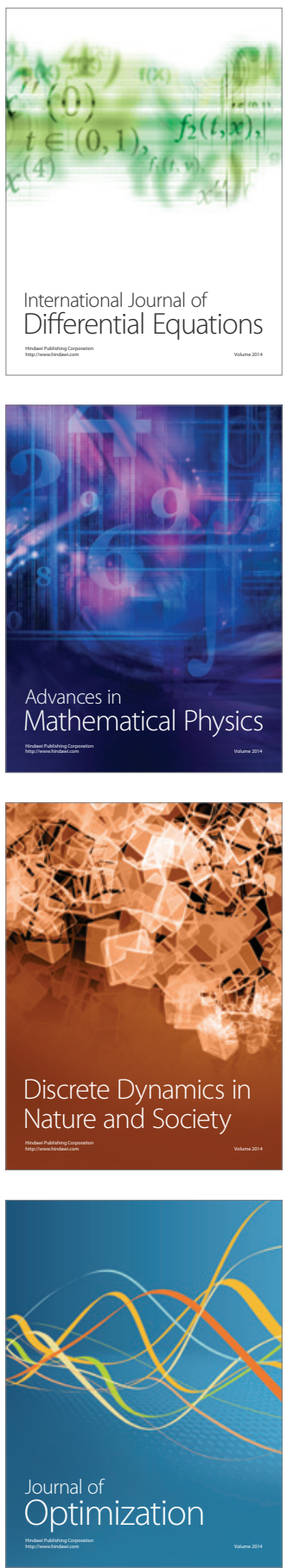